\title{
A Criterion for Using Chloramphenicol to Define Different Processes in the Initiation of DNA Synthesis in Bacteria
}

\author{
STEPHEN COOPER \\ Department of Microbiology, \\ The University of Michigan Medical School, \\ Ann Arbor, Michigan 48104, U.S.A.
}

(Received 14 May 1973, and in revised form 21 September 1973)

\begin{abstract}
A criterion is proposed which must be met in order to demonstrate that two different proteins are required for the initiation of DNA synthesis, with each protein being synthesized at a different time prior to initiation, and with the synthesis of each protein having a different sensitivity to inhibition by chloramphenicol. Specifically, as the CM concentration is continuously varied, there must be a discontinuous variation in the number of origins able to be initiated at each concentration. In the light of this criterion, I suggest that there is no firm evidence for the proposal that there exist two different proteins which are required for initiation of DNA replication in Escherichia coli.
\end{abstract}

\section{Introduction}

It has been suggested (Lark \& Renger, 1969; Ward \& Glaser, 1969; Messer, 1972) that there are two proteins required for the initiation of a round of DNA replication in Escherichia coli, and that the synthesis of these two proteins have different sensitivities to inhibition by chloramphenicol (CM), a compound which inhibits protein synthesis. Specifically, it has been suggested that there is a protein normally synthesized a few minutes prior to initiation, whose synthesis is inhibited by "high" concentrations of CM but not by "low" concentrations of $\mathrm{CM}$. There is a suggested second protein whose synthesis is sensitive to both "high" and "low" concentrations of CM and which is normally synthesized somewhat earlier than the first protein. (The actual "high" and "low" concentrations, as well as the proposed timing, depend on the strain and the experimental conditions.)

I wish to re-examine the evidence supporting this idea, and to explicitly state a criterion which must be met before the above conclusions can be made. I suggest that the criterion described below has not been met. 


\section{A Criterion for Demonstrating Differential Sensitivity of Initiator Proteins}

Consider cells incubated in very "high" concentrations of CM. Cells which have completed both postulated proteins will be able to initiate new rounds of replication. Now, consider a series of cultures to which different and continuously decreasing concentrations of $\mathrm{CM}$ are added. If two distinct steps did exist, it would be expected that a concentration would eventually be reached which would barely inhibit the synthesis of the protein which was sensitive only to the "high" concentrations of CM. As the concentration was lowered slightly, those cells which had already synthesized the protein sensitive to both "high" and "low" concentrations of CM, would now be able to synthesize the protein which is sensitive only to the "high" concentration of CM. Thus a new group of cells would be able to initiate new rounds of replications in the presence of $\mathrm{CM}$. The exact number of cells allowed to proceed to initiate new rounds of replication would depend on the difference in the time when the proteins were normally synthesized. As the concentrations of $\mathrm{CM}$ were further lowered a concentration would be reached which was just above the concentration which would inhibit the process sensitive to "low" (and "high") concentrations of CM. When this limit was passed, and the concentration of CM went below the postulated "low" concentration, there would be a new group of cells which would be able to initiate rounds of replication in the presence of the inhibitor. The expected variation in initiation in the presence of $\mathrm{CM}$, as a function of $\mathrm{CM}$ concentration, is illustrated by the heavy line in Fig. 1. If the two protein model were correct, as the con-

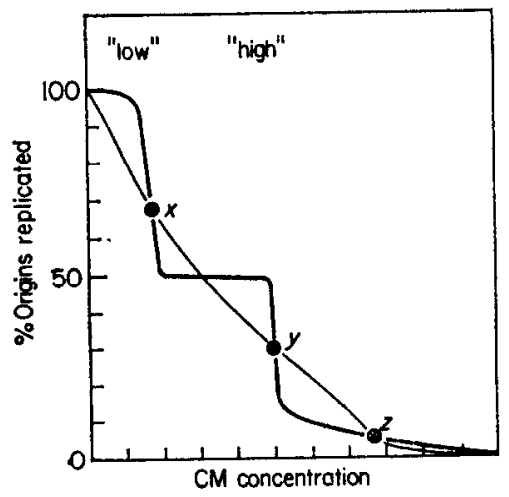

Fic. 1. Idealized representation of the variation in number of origins replicated in the presence of CM assuming that either (a) the model proposed by Lark \& Renger is correct (thick line), or (b) that leakiness of protein synthesis occurs over the range of CM concentrations tested (thin line). Points $x, y$, and $z$ are discussed in the text. 
centration of $\mathrm{CM}$ was continuously lowered, stepwise increases in the number of origins allowed to replicate in the presence of $\mathrm{CM}$ at both the proposed "high" and "low" concentrations of CM would be predicted.

If a limited number of measurements were made, for example points $x, y$, and $z$ in Fig. 1, the results would be indistinguishable from the thin line which fits the same points. The reason that measurements must be taken over a continuous range of $\mathrm{CM}$ concentrations is to eliminate this possible result. What conclusions could be made if the results fit the thin, rather than the thick line of Fig. 1 ? What possible explanations would there be if there was a continuous increase in the number of chromosomes allowed to initiate new rounds of replication in the presence of $\mathrm{CM}$ as the $\mathrm{CM}$ concentration was lowered? I suggest three possibilities which can fit this result as follows:

(1) different steps do exist, analogous to the two steps previously postulated, but there are actually an enormous number of steps, each one defined by a different concentration of chloramphenicol; or

(2) there are no distinct CM definable steps, and the different amounts of initiation are actually due to different amounts of "leakage" of protein synthesis in the presence of limiting amounts of $\mathrm{CM}$; or

(3) there are actually only two different steps in the initiation of DNA synthesis, as has been postulated, but the probability of inhibition by a given concentration of $\mathrm{CM}$ has a wide dispersion which obscures the predicted steps. In this case, one would have to conclude that it would not be possible to experimentally observe the predicted steps of Fig. 1.

In the light of the above discussion, the criterion for demonstrating different steps in initiation by differential $C M$ sensitivity is: as the concentration of $C M$ is lowered continuously, there must be stepwise increases in the number of initiating origins at each postulated concentration. A continuous increase would be consistent with a number of other interpretations.

I will now analyze the data presented for and against the main proposal.

\section{The Experiments of Lark \& Renger}

Before examining the data of Lark \& Renger (1969), I will explain the nature of the experiments they performed. In a simplified form, the experiment is as follows. Cells were starved of amino acids to allow terminalization of all chromosomes in the process of replication, and then replication of chromosomes in the presence of amino acids and radioactive thymine was allowed to occur. After a short period of labeling, when only the origins of the chromosomes were labeled, the radioactivity was removed, and amino acid starvation 
continued to terminalize chromosomes. At this time (zero minutes), amino acids were replaced and the cells were grown in a medium with $\mathrm{C}^{\mathbf{1 3}}$-glucose and $\mathrm{N}^{15} \mathrm{H}_{4} \mathrm{Cl}$ as density labels. One culture was continuously incubated and sampled at different times for the amount of radioactive DNA (origin DNA) which was in a hybrid form (by $\mathrm{CsCl}$ centrifugation). This is plotted in Fig. 2

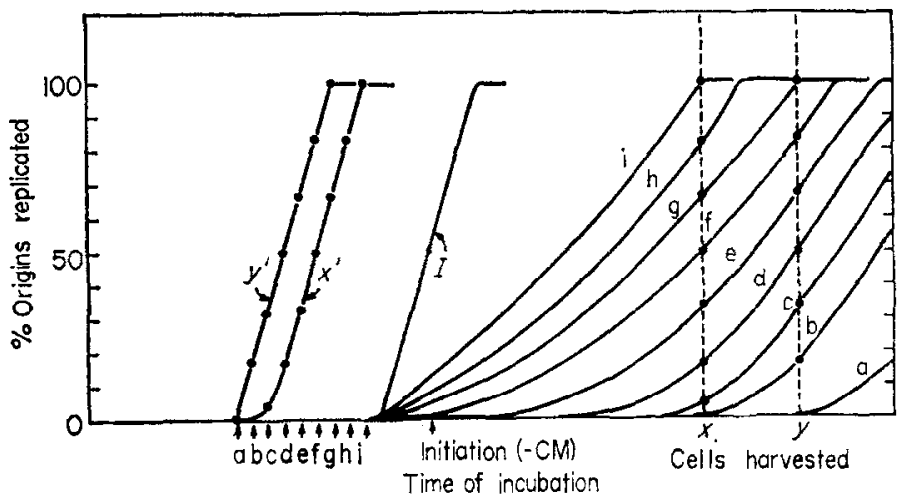

Fig. 2. Idealized representation of the experiments of Lark \& Renger assuming that leakage accounts for the observed results. Lines $x^{\prime}$ and $y^{\prime}$ are derived by replotting the points observed at times $x$ and $y$ at the time that the CM was added. For example, life f generates the two points (at intersection with lines $x$ and $y$ ) which are plotted above time $f$ in lines $x^{\prime}$ and $y^{\prime} . I$ is the line indicating the normal time that initiation takes place in the culture in the absence of any $\mathrm{CM}$. Lines a- $\mathrm{i}$ are the predicted time course of initiation assuming that leakiness accounts for the results.

as line $I$. This represents the time course of chromosome initiations occurring in the absence of chloramphenicol. Imagine that at zero minutes aliquots of the same culture are put into a number of different flasks, and a constant amount of $\mathrm{CM}$ is added at different times (a-i). Incubation of each of these cultures is now continued until some later time, $x$, at which the cells are harvested, lysed, and analyzed for the amount of radioactive DNA in hybrid form. Imagine that the inidividual points on line $x$ of Fig. 1 were actually obtained. This is plotted on the right as line $x^{\prime}$, representing the amount of origin DNA which can be replicated in the presence of $\mathrm{CM}$ when the $\mathrm{CM}$ is given at the indicated time (a-i). The difference between the lines $x^{\prime}$ and $I$ would be interpreted by Lark \& Renger as the minutes prior to actual initiation, that a protein was synthesized, whose synthesis was sensitive to the given concentration of chloramphenicol. If the points on line $x$ were actually generated by a general "leakage" of protein synthesis, the lines in Fig. 2, labeled a-i (corresponding to the times of addition of $\mathrm{CM}, \mathrm{a}-\mathrm{i}$ ), could be the actual time course of initiation in the presence of $\mathrm{CM}$. This result is plausible 
assuming "leakage" as the earlier the CM is added the more "leakage" must occur to make the protein(s) that are involved in initiation. However, if the cells would have been harvested at a different and later time, $y$, a different set of points would be obtained. When these points were replotted on the right with the associated time of addition of CM, they would generate line $y^{\prime}$. Thus, if "leakage" was a factor, the time at which cells were actually harvested would be a determining factor in estimating when the protein, sensitive to the given concentration of $\mathrm{CM}$, was synthesized. The later the harvesting took place, the earlier one would conclude that the protein sensitive to CM was synthesized.

If the model of Lark \& Renger were correct, the data of line $x$ would be generated by the lines a-i of Fig. 3. That fraction of cells which had actually

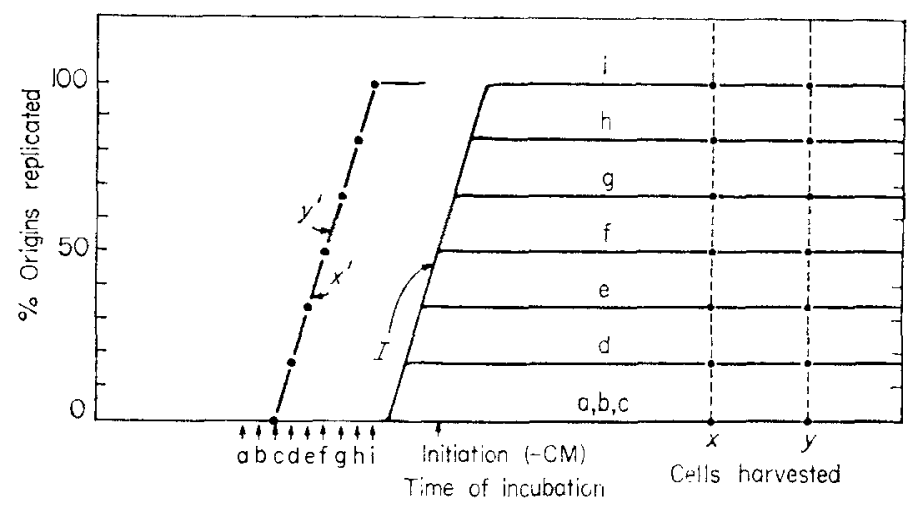

FIG. 3. Idealized representation of the experiments of Lark \& Renger assuming that their model is correct. The general plan of the figure is similar to that of Fig. 2.

synthesized the required protein would initiate DNA synthesis at the time initiation would normally occur. No further initiations would occur during prolonged incubation. The data points of line $y$ would be identical to line $x$; lines $x^{\prime}$ and $y^{\prime}$ would be identical (Fig. 3).

Lark \& Renger (1969) have presented an experiment (their Fig. 5) in which samples were taken at different times from a culture growing in dense medium. They have measured a line similar to a-i (Fig. 2 or 3 ) to determine the actual time course. There is no plateau observed. I suggest that the time at which cells are harvested does have an effect on the observed time at which one concludes the inhibition by CM has been overcome. Further, this experiment of Lark and Renger is consistent with "leakage" as described in Fig. 2.

The concentration of $\mathrm{CM}$ used can also change the observed time at which sensitivity to CM is overcome. This is illustrated in Fig. 4. If "leakage" is a 


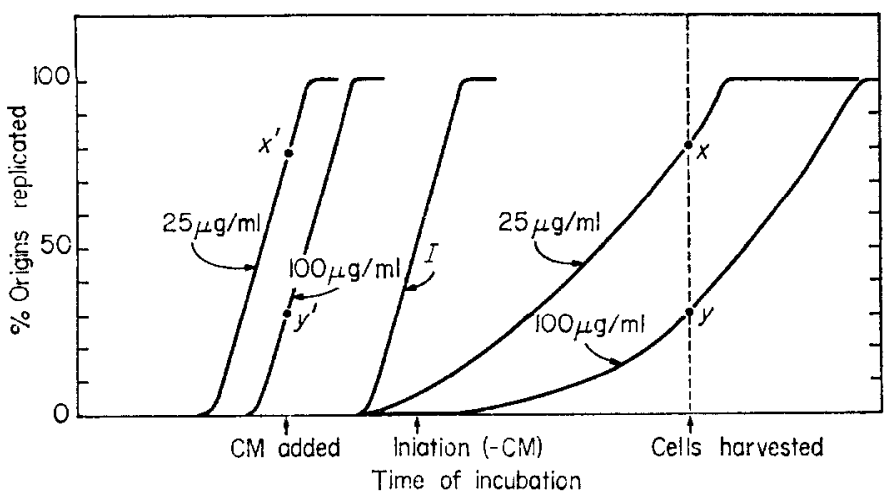

FIG. 4. Idealized representation of the experiments of Lark \& Renger demonstrating the effect of different concentrations of $\mathrm{CM}$ on the observed time at which $\mathrm{CM}$ inhibition is bypassed, assuming that leakiness can account for the results.

factor, one might expect two curves as drawn for 25 and $100 \mu \mathrm{g} / \mathrm{ml}$ of $\mathrm{CM}$. Note that each curve (along with other curves not shown) would lead to the lines determined by points $x$ and $y$. Here one would conclude that the synthesis of a protein sensitive to $25 \mu \mathrm{g} / \mathrm{ml}$ occurred somewhat earlier than the synthesis of a protein sensitive to $100 \mu \mathrm{g} / \mathrm{ml}$ of $\mathrm{CM}$. Considering the criterion stated

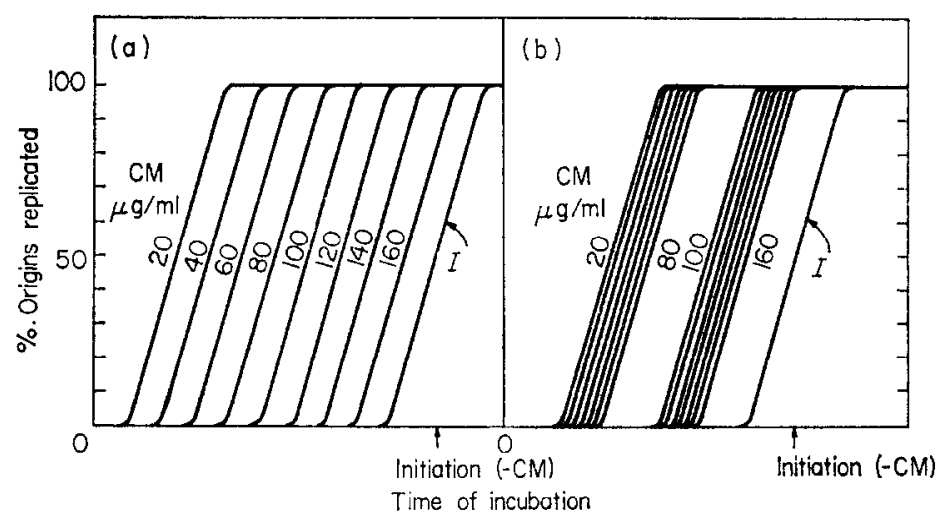

FIG. 5. (a) Predicted results of the experiments of Lark \& Renger if "leakage" can account for their experimental results. Rather than being taken literally, that there should be equal distances between the lines as the concentration is varied, the main point is that there should be no indication of a discontinuity, or stepwise increase in the ability to initiate new origins as the concentration is lowered. (b) Predicted results of the experiments of Lark \& Renger assuming that their model is correct. This figure would indicate that there was a concentration (approximately $90 \mu \mathrm{g} / \mathrm{ml}$ ) below which a discrete number of new origins would be able to be initiated, and above which they would not be initiated. This result would be equivalent to the thick line of Fig. 1. 
above, one would have to distinguish two possibilities as illustrated in Fig. 5. Either there is a continuous variation, with decreasing $\mathrm{CM}$ concentration, of the number of origins allowed to initiate (a), or there is a discontinuous variation in the number of origins allowed to initiate as the $\mathrm{CM}$ concentration decreases (b). These two possibilities are analogous to the heavy line in Fig. 1. Since Lark \& Renger (1969) studied only two concentrations (analogous to points $x$ and $y$ in Fig. 1 or the two lines $x^{\prime}$ and $y^{\prime}$ in Fig. 4), the criterion proposed above has not been met, and their results are also consistent with other possible models as described above.

Lark (1973) has attempted to demonstrate the proposed discontinuity by investigating $50 \mu \mathrm{g} / \mathrm{ml} \mathrm{CM}$ [Lark \& Renger (1969) studied 25 and $150 \mu \mathrm{g} / \mathrm{ml}$ $\mathrm{CM}$. He suggests that the results with 50 and $25 \mu \mathrm{g} / \mathrm{ml} \mathrm{CM}$ are identical. However, Lark compared his results to an experimental line from Lark \& Renger (1969). One culture was not investigated over a range of CM concentrations.

In order to clarify the problem, I will explicitly state the experiment which would be able to prove the model of Lark \& Renger (1969). That is, on one day, with one culture, a wide range of different $\mathrm{CM}$ concentrations must be added to different aliquots at different times and incubation continued and all flasks harvested at one final time. The results ( $\%$ origins replicated) can then be plotted to see whether the results fit Fig. 5(a) or (b).

\section{The Experiments of Ward \& Glaser}

Ward \& Glaser (1969) used a different approach, and used a different strain of bacteria, $E$. coli $\mathrm{B} / \mathrm{r}$, but their experiments were designed merely to extend the model of Lark \& Renger (1969) to E. coli $\mathrm{B} / \mathrm{r}$, and to determine the concentrations of $\mathrm{CM}$ analogous to those proposed for $E$. coli $15 \mathrm{~T}^{-}$. The model was not tested according to the criterion proposed above. They concluded that instead of the 25 and $150 \mu \mathrm{g} / \mathrm{ml}$ concentrations found in $15 \mathrm{~T}^{-}$the two proteins in $E$. coli $\mathrm{B} / \mathrm{r}$ were sensitive to 2 and $30 \mu \mathrm{g} / \mathrm{ml}$ of CM respectively. No continuous range of concentrations were investigated.

\section{The Experiments of Messer}

Messer (1972) reinvestigated the results of Ward \& Glaser (1969) with strain B/r and also assumed that the model of Lark \& Renger (1969) was essentially correct. He was primarily interested in determining the timing of the two postulated events. Ward \& Glaser (1969) had proposed that in strain $\mathrm{B} / \mathrm{r}$ the second protein was synthesized coincident with initiation, whereas 
Messer proposed a timing situation more analogous to Lark \& Renger. Again, there was no attempt to test a wide range of concentrations in his primary experimental situation, synchronized cells.

One experiment by Messer is of interest. In it he looked at the effect of six concentrations of CM on the rate of DNA synthesis in an exponentially growing culture. I will restate the proposal of Messer, and then compare this proposal with his experimental results.

Messer's proposal, which may be divided into two forms, is illustrated in Fig. 6. He postulated that one process occurring $20 \mathrm{~min}$ prior to initiation is inhibited by $2 \mu \mathrm{g} / \mathrm{ml}$ of $\mathrm{CM}$. A second step, occurring 5-10 min prior to initiation is sensitive to $4-30 \mu \mathrm{g} / \mathrm{ml}$ of $\mathrm{CM}$. Figure 6 illustrates the idea that

\begin{tabular}{|c|c|c|}
\hline & $10 \mathrm{~min}$ & $5-10 \mathrm{~min}$ \\
\hline$(a)$ & Sensitive to $2 \mu \mathrm{g} / \mathrm{ml}$ & $\begin{array}{l}\text { Sensitive to } 4-30 \mu \mathrm{g} / \mathrm{ml} \\
\text { Resistont to }<4 \mu \mathrm{g} / \mathrm{ml}\end{array}$ \\
\hline (b) & Sensitive to $2 \mu \mathrm{g} / \mathrm{ml}$ & $\begin{array}{l}\text { Sensitive to } 4-30 \mu \mathrm{g} / \mathrm{ml} \\
\text { Sensitive to }>2 \mu \mathrm{g} / \mathrm{ml}\end{array}$ \\
\hline
\end{tabular}

Fig. 6. The model of Messer for $E$. coli $\mathrm{B} / \mathrm{r}$. Two possible interpretations of his model are presented. These are indicated diagrammatically in Fig. 7.

above $4-30 \mu \mathrm{g} / \mathrm{ml}$ of $\mathrm{CM}$ all cells which are within $5-10 \mathrm{~min}$ of initiation will initiate new rounds of replication in the presence of $\mathrm{CM}$. Those further away in time from initiation will not initiate because the synthesis of some protein is sensitive to these concentrations of CM. Therefore, the residual DNA synthesis will be due to completion of chromosomes in progress, and completion of chromosomes in these cells within 5-10 min of initiation. Model (a) further suggests that below $4 \mu \mathrm{g} / \mathrm{ml}$ of $\mathrm{CM}$ all cells within $10-20 \mathrm{~min}$ prior to initiation will now also initiate new rounds of replication in addition to those that would have initiated new rounds of replication with concentrations above $4 \mu \mathrm{g} / \mathrm{ml} \mathrm{CM}$. Model (b) on the other hand, states that only when $2 \mu \mathrm{g} / \mathrm{ml}$ or less of chloramphenicol is present will the additional cells which are within $10-20 \mathrm{~min}$ of initiation be able to initiate new rounds of replication.

Figure 7 is the data of Fig. 4 of Messer (1972) replotted. In this experiment Messer measured the rate of DNA synthesis in exponentially growing cells, at different times in the presence of six different concentrations of CM. Figure 7 uses the rate of DNA synthesis at $80 \mathrm{~min}$ after adding the CM. Since the rate of DNA synthesis decreased exponentially in this experiment (an unexpected observation) the choice of $80 \mathrm{~min}$ is equivalent to any other time. The predicted results expected for models (a) and (b) are also indicated. There is no 


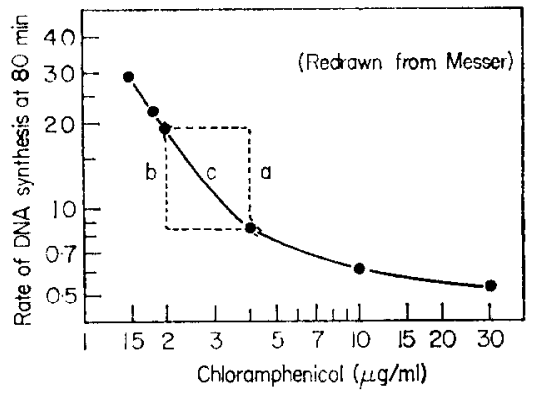

FIG. 7. Replotting of the data of Fig. 4 of Messer (1972). The dashed lines a and $b$ are the predicted results assuming the models $a$ and $b$ of Fig. 6 . Now shown, but theoretically expected, is another plateau in line $b$, just to the left of $2 \mu \mathrm{g} / \mathrm{ml}$, before the line again increases.

indication that the experimental curve follows model (a) or (b). That a plateau (or discontinuity) as illustrated in curves $\mathbf{a}$ or $\mathbf{b}$ is experimentally observed is a necessary criterion for the hypothesis that two steps, separable by differential sensitivity to $\mathrm{CM}$ exists. If, for example, an intermediate rate was found with $3 \mu \mathrm{g} / \mathrm{ml}$ of CM, that would define an additional step, different from the other two processes, which occurred approximately 15 min prior to initiation, and which is sensitive to $3 \mu \mathrm{g} / \mathrm{ml} \mathrm{CM}$.

\section{The Experiments of Cooper \& Wuesthoff}

Cooper \& Wuesthoff (1971) investigated the synthesis of DNA in the presence of different amounts of $\mathrm{CM}$ in both $E$. coli $\mathrm{B} / \mathrm{r}$ and $15 \mathrm{~T}^{-}$. They observed a continuum in the amount of residual DNA synthesis as the concentration was lowered. The concentration range over which the residual DNA synthesis increased with decreasing $\mathrm{CM}$ concentration was similar to the proposed concentration steps in the two strains. Their experiments were directed toward showing that the criterion proposed above has not been met. Further, they showed that a decreased inhibition of DNA synthesis as the CM concentration was lowered, was paralleled by a similar decrease in the inhibition of protein synthesis. That is, the results of Lark \& Renger (1969), Ward \& Glaser (1969), and Messer (1972) can be accounted for by lowered inhibition of protein synthesis as the CM concentration is lowered. Messer (1972) reported that at $2 \mu \mathrm{g} / \mathrm{ml}$ of CM, there is $10 \%$ residual protein synthesis, and Ward \& Glaser reported $15 \%$ residual protein synthesis with $2 \mu \mathrm{g} / \mathrm{ml}$ CM. This suggests that "leakage" can explain the data demonstrating two different proteins involved in initiation of DNA replication. 


\section{Does CM Affect Elongation of the Chromosome?}

Messer (1972) and Lark (1973) have criticized the results of Cooper \& Wuesthoff (1971). They suggest that CM also affects the elongation of the chromosome. If this were the case, then it would be a factor in the interpretation of the residual DNA synthesis results of Cooper \& Wuesthoff (1971). I will re-examine the evidence presented by Messer and by Lark. I will suggest that there is no firm evidence that $\mathrm{CM}$ affects chromosome elongation. This is not to say that it does not affect elongation but that there is no firm evidence which would lead to the discarding of the assumption that $\mathrm{CM}$ allows rounds of replication in progress to go to completion.

Messer (1972) does not actually prove this point; he accepts it as shown by Lark (1973), and suggests that various dips in his incorporation curves are consistent with the idea. As the proposal is not precisely presented it is not clear what would be expected to be observed in the experiments of Messer. Also, if CM does affect elongation, then Messer's results are the combination of two effects which cannot be distinguished. That is, his results are very much dependent upon the assumption that rounds of replication, once started, go unhindered to completion in the presence of $\mathrm{CM}$.

The experiment of Lark (1973) is more to the point. The experiment he reports which bears on this problem is a measurement of the amount of DNA synthesis in the presence of different concentrations $(0,20,30,40$, and $50 \mu \mathrm{g} / \mathrm{ml}$ ) of $\mathrm{CM}$. Lark labeled chromosomes completely with $\mathrm{C}^{14}$ thymine and they were then replicated to the middle of a round of replication. The medium was changed to a dense label $\left(\mathrm{C}^{13}-\mathrm{N}^{15}\right)$ and after $100 \mathrm{~min}$ of incubation, the amount of DNA in hybrid form was determined. Lark observed a decreasing amount of hybrid radioactivity as the concentration of CM increased. Lark concluded that "the assumption that replication can be completed in chloramphenicol is incorrect and the amount of residual replication is dependent on the concentration of the drug". This conclusion may be correct only if variation in CM concentration does not affect initiation, but as has been noted above, this is not yet proven. Lark (1973) does suggest that over the range of concentrations studied by him initiation is not affected. However, as mentioned above, this conclusion is drawn from a comparison of two experiments performed on different days. Therefore, the results of Lark (1973) can also be explained by a differential sensitivity of initiation to CM. The variation in DNA synthesis can be due to a variation in the degree of initiation. Since Lark incubated the cells $100 \mathrm{~min}$, and initiation would have been expected to occur in $20 \mathrm{~min}, 80 \mathrm{~min}$ of continued incubation could allow enough "leakage" of protein synthesis and the associated initiation, to account for the results. I therefore suggest that more 
experiments are needed to demonstrate that $\mathrm{CM}$ affects the completion of rounds of replication in progress. For example, the experiment of Lark (1973) should be repeated with the replication of labeled termini in various CM concentrations determined directly. This would avoid the complication of variation of initiation in different $\mathrm{CM}$ concentrations.

This work has been supported by a Public Health Service Grant AI-10059.

\section{REFERENCES}

COOPER, S. \& Wuestoff, G. (1971). J. Bact. 106, 709.

LARK, K. G. (1973). J. Bact. 113, 1066.

LARK, K. G. \& ReNGer, H. (1969). J. molec. Biol. 42, 221.

MEsSER, W. (1972). J. Bact. 112, 7.

WARD, C. G. \& GlaSER, D. (1969). Proc. natn. Acad. Sci. U.S.A. 64, 905. 